\title{
LUMINESCENCE PROPERTIES OF EUROPIUM IN SORBATES OF THE COMPLEXES WITH NAPHTHOIC ACID ON ZEOLITE*
}

\author{
S. V. BELTYUKova
}

Odessa State Academy of Food Technologies. Dep. of Anal. Chem. 112, Kanatnaya St., 270030, Odessa, Ukraine

\author{
A.V. Egorova, E.I. Tselic and O.I. Teslyuk
}

A.V. Bogatsky Physico-Chemical Institute of the National Academy of Sciences of Ukraine, 86, Lustdorfskaya doroga, 270080, Odessa, Ukraine

(Received July 23, 1998; revised version February 25, 1999)

A study of luminescence properties of sorbates of the europium(III) complexes with naphthoic acid and 1,10-phenanthroline on the zeolite of CaA-type that may be used as luminophores of the red color luminosity, has been performed. It was shown that the highest luminescence intensity in sorbate is observed upon the preliminary sorption of $\mathrm{Eu}(\mathrm{III})$ ion on zeolite following the treatment of naphthoic acid and 1,10-phenanthroline. The stability of sorbates to the temperature action was established $\left(100^{\circ} \mathrm{C}, 2 \mathrm{~h}\right)$.

PACS numbers: $78.55 . \mathrm{Cr}$

\section{Introduction}

In recent years the time lanthanide complexes with organic ligands attract the attention of many investigators because in these compounds the intramolecular energy transfer from ligand triplet levels to lanthanide emitting levels is possible. Therefore, they are able to transform the short wave radiation of mercury $(185 \mathrm{~nm}$ and $254 \mathrm{~mm}$ ) into the visible one [1-3]. Now such luminophores based on the terbium(III) complex with benzoic acid incorporated into the zeolite lattice has been proposed as promising materials for the lamp luminophores $[4,5]$. The formed terbium benzoate possesses the intensive luminescence at $545 \mathrm{~nm}$, the quantum yield of luminescence of this phosphor is about $50 \%[4,5]$. The introduction of complex into the matrix of zeolite stimulates a reduction in the expense of rare earth element for preparation of luminophore material and, consequently, a decrease in its price [4].

The purpose of this work is the study of optimum conditions of luminescence of europium(III) complex with naphthoic acid and 1,10-phenanthroline sorbed on the zeolite. The luminescence properties of europium(III) ion in toluol extracts

*The results of thiis paper were initially presented at The Jabloniski Centennial Conference on Luminescence and Photophysics, July 23-27, 1998, Torun, Poland. 
of complexes with the above-mentioned ligands have been previously investigated [6]. It has been supposed that the introduction of this complex into the lattice of zeolite, i.e. its fastening on solid surface will stimulate a decrease in the non-radiative energy losses and, consequently, an increase in the luminescence intensity of $\mathrm{Eu}(\mathrm{III})$.

\section{Experimental}

\subsection{Apparatus}

The luminescence measurements were recorded on an SDL-2 spectrophotometer (Leningrad Opto-Mechanical Association, St. Petersburg, Russia). A xenon lamp was used as an excitation source. The $\mathrm{pH}$ values of solutions were measured. using an OP-211/1 laboratory digital pH-meter (Radelkis, Budapest, Hungary).

\subsection{Reagents}

The europium(III) chloride solution with a concentration of $0.1 \mathrm{~mol} / \mathrm{L}$ prepared by dissolving the europium oxide (99.99\%) in hydrochloric acid (1:1) following evaporation of acid excess and dissolution with distilled water was used. The metal concentration was established by complexometric with Arsenazo I. The aqueous solution of 1,10-phenanthroline $(0.1 \mathrm{~mol} / \mathrm{L})$ and ethanolic solution of naphthoic acid $\left(4 \times 10^{-2} \mathrm{~mol} / \mathrm{L}\right)$ were obtained by dissolution of accurately weighed preparations in distilled water upon acidifying and ethanol, respectively. The $\mathrm{pH}$ values was adjusted with $0.2 \mathrm{~mL}$ of $8 \%$ solution of urothropine. The zeolite of CaA mark was preliminary sited through the sieve with the aperture diameter of $0.1 \mathrm{~mm}$. The zeolite fraction with a particle size less than $0.1 \mathrm{~mm}$ was washed with hydrochloric acid for $2 \mathrm{~h}$, upon the stirring, washed with distilled water to neutral reaction and dried at a temperature of $100^{\circ} \mathrm{C}$ for $2 \mathrm{~h}$.

\subsection{Methods}

To choose the optimum conditions of the sorbates luminescence the assays were prepared as the following. The sorption of europium ions preliminary was performed on the zeolite, to obtain the complex compound, the zeolite was treated with naphthoic acid (NP) and 1,10- phenanthroline (Phen) solutions at an appropriate $\mathrm{pH}$ value.

Then the zeolite was filtered off, washed with aqueous-ethanolic mixture (1:1), dried at a temperature of $80^{\circ} \mathrm{C}$ for $2-3 \mathrm{~h}$. Luminescence of sorbates was recorded at $612 \mathrm{~nm}$. The sorption was performed in static conditions.

\section{Results and discussion}

\subsection{Optical characteristics of ligand and sorbates}

The naphthoic acid and 1,10-phenanthroline were taken as the ligands effectively absorbing the excitation energy. The absorption spectrum of naphthoic acid in ethanolic solution possesses the intensive absorption band in a visible spectral region at $225 \mathrm{~nm}$, the molar extinction coefficient of which is $9 \times 10^{4}$. In aqueous solution of 1,10-phenanthroline there are two absorption bands at $232 \mathrm{~nm}$ and $262 \mathrm{~nm}$ and the molar extinction coefficients for these bands are $7 \times 10^{4}$ and $4 \times 10^{4}$, 


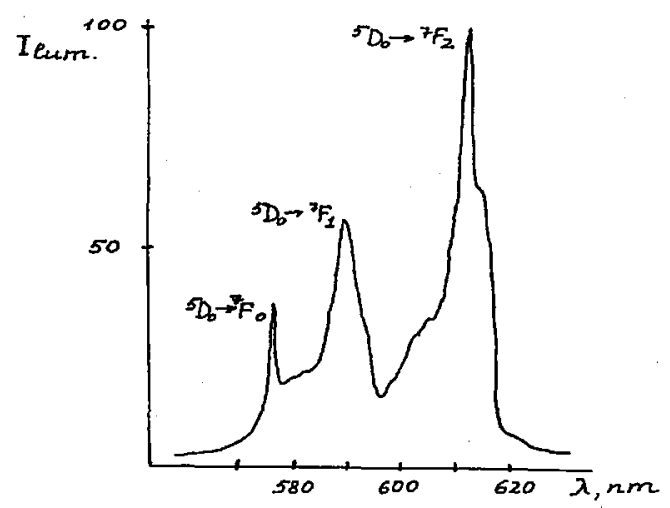

Fig. 1. The luminescence spectrum of sorbate of $\mathrm{Eu}(\mathrm{III})$ complex with naphthoic acid and 1,10-phenanthroline on the zeolite.

respectively. The high values of molar extinction coefficients stimulate effective absorption of light energy by the ligands that is transferred to the europium ion. Three bands corresponding to ${ }^{5} D_{0} \rightarrow{ }^{7} F_{2}(\lambda=612 \mathrm{~nm}),{ }^{5} D_{0} \rightarrow{ }^{7} F_{1}(\lambda=590 \mathrm{~nm})$ and ${ }^{5} D_{0} \rightarrow{ }^{7} F_{0}(\lambda=580 \mathrm{~nm})$ transitions are observed in luminescence spectrum of sorbate of europium complex (Fig. 1). The distribution of intensity in these bands is the following:

$$
\begin{array}{lr}
\text { Transition } & I_{\text {lum }} \% \\
{ }^{5} D_{0} \rightarrow{ }^{7} F_{2}, & 100, \\
{ }^{5} D_{0} \rightarrow{ }^{7} F_{1}, & 55, \\
{ }^{5} D_{0} \rightarrow{ }^{7} F_{0}, & 35 .
\end{array}
$$

The most intensive is the band corresponding to hypersensitive transition ${ }^{5} D_{0} \rightarrow{ }^{7} F_{2}$.

\subsection{Influence of the sorption time and europium concentration}

The luminescence intensity of the sorbates depends on the sorption time and concentration of europium(III) ion in sorbates.

The europium ions were introduced into the cavities of zeolite through the ionic exchange.

The sorption time of Eu(III) ion was studied as the following: $0.5 \mathrm{~mL}$ of $0.1 \mathrm{~mol} / \mathrm{L}$ solution of $\mathrm{Eu}$ (III) chloride was introduced into the glass, $\mathrm{pH}$ of solution was adjusted to $2-3,60 \mathrm{mg}$ of zeolite was added, solution was diluted with water and stirred for different time (30 min, 1,2,3,4,5, and $7 \mathrm{~h}$ ). Then the zeolite was filtered out, washed with aqueous-ethanolic mixture (1:1) and dried. The dried zeolite was treated by naphthoic acid and 1,10-phenanthroline solutions, filtered again, washed and dried. The luminescence intensity in sorbates is presented in Fig. 2. As can be seen, the optimum sorption time of europium from solutions is $5 \mathrm{~h}$. The luminescence intensity in sorbates depends on the amount of europium(III) ions introduced into the zeolite cavities. As can be seen from Fig. 3, the highest $I_{\text {lum }}$ in sorbates is observed at the concentration of $\mathrm{Eu}(\mathrm{III}) 0.1 \mathrm{~mol} / \mathrm{L}$. 


\section{Fig. 2}
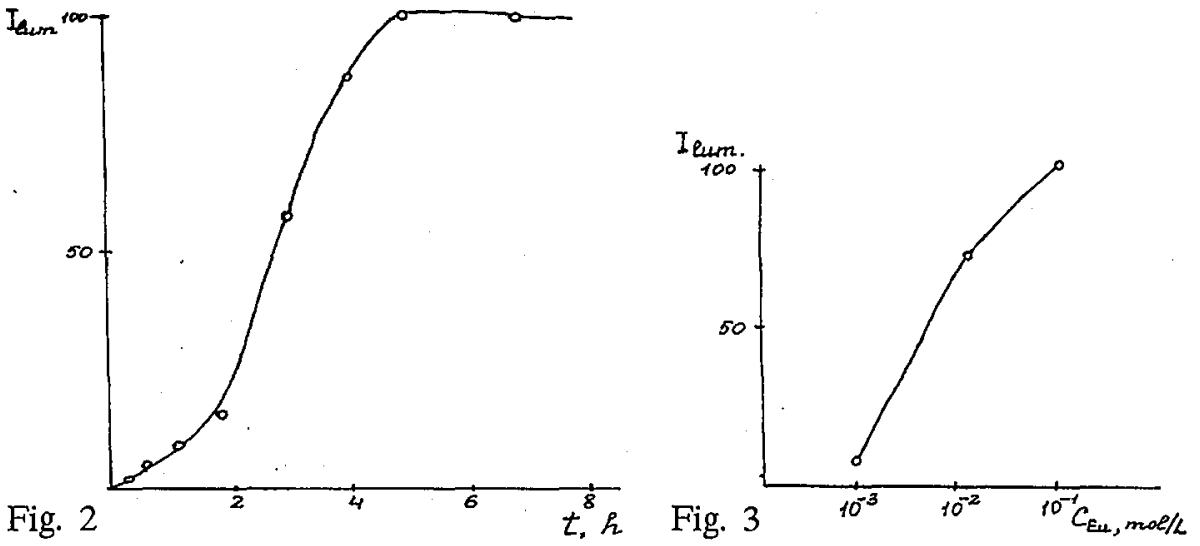

Fig. 2. Dependence of $I_{\text {lum }}$ in sorbates of the complex on sorption time of europium on zeolite; $C_{\mathrm{Eu}}=1 \times 10^{-2} \mathrm{~mol} / \mathrm{L}, C_{\mathrm{NP}}=2.5 \times 10^{-2} \mathrm{~mol} / \mathrm{L}, C_{\mathrm{Phen}}=2.0 \times 10^{-2} \mathrm{~mol} / \mathrm{L}$. Fig. 3. Dependence of $I_{\mathrm{lum}}$ of $\mathrm{Eu}(\mathrm{III})$ in sorbates of complexes on $\mathrm{Eu}(\mathrm{III})$ amount in sorbate; $C_{\mathrm{NP}}=2.5 \times 10^{-2} \mathrm{~mol} / \mathrm{L}, C_{\text {Phen }}=2.0 \times 10^{-2} \mathrm{~mol} / \mathrm{L}$.

\subsection{Influence of $\mathrm{pH}$}

The dependence of $I_{\text {lum }}$ of $\mathrm{Eu}$ (III) in sorbate on $\mathrm{pH}$ of aqueous phase, from which the sorption of complex was performed, was studied. It was shown that the highest $I_{\text {lum }}$ of $\mathrm{Eu}(\mathrm{III})$ is observed in a range of $\mathrm{pH} 6.0-7.0$ with a maximum at $\mathrm{pH}=6.5$ (Fig. 4) corresponding to the $\mathrm{pH}$ value for the optimum complex formation in solution [6].

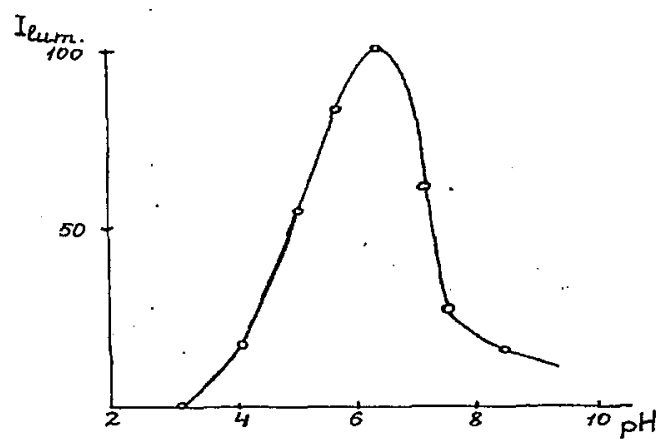

Fig. 4. Dependence of $I_{\text {lum }}$ of Eu(III) in sorbate of complex on $\mathrm{pH}$ of aqueous phase; $C_{\mathrm{Eu}}=3 \times 10^{-4} \mathrm{~mol} / \mathrm{L}, C_{\mathrm{NP}}=2.5 \times 10^{-2} \mathrm{~mol} / \mathrm{L}, C_{\mathrm{Phen}}=2.0 \times 10^{-2} \mathrm{~mol} / \mathrm{L}$.

\subsection{Influence of the sorption time and ligand concentration}

It was preliminary established that 15 min of stirring the zeolite with naphthoic acid and 1,10-phenanthroline solutions is enough to obtain the highest $I_{\text {lum }}$ of $\mathrm{Eu}(\mathrm{III})$ in sorbates (Fig. 5). 


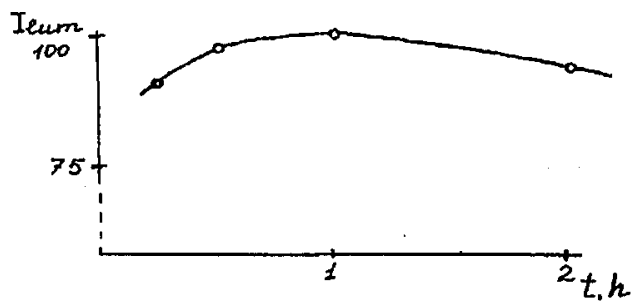

Fig. 5. Dependence of $I_{\text {lum }}$ of $\mathrm{Eu}$ (III) in sorbate of complex on the sorption time of ligands; $C_{\mathrm{Eu}}=1 \times 10^{-2} \mathrm{~mol} / \mathrm{L}, C_{\mathrm{NP}}=2.5 \times 10^{-2} \mathrm{~mol} / \mathrm{L}, C_{\text {Phen }}=2.0 \times 10^{-2} \mathrm{~mol} / \mathrm{L}$.
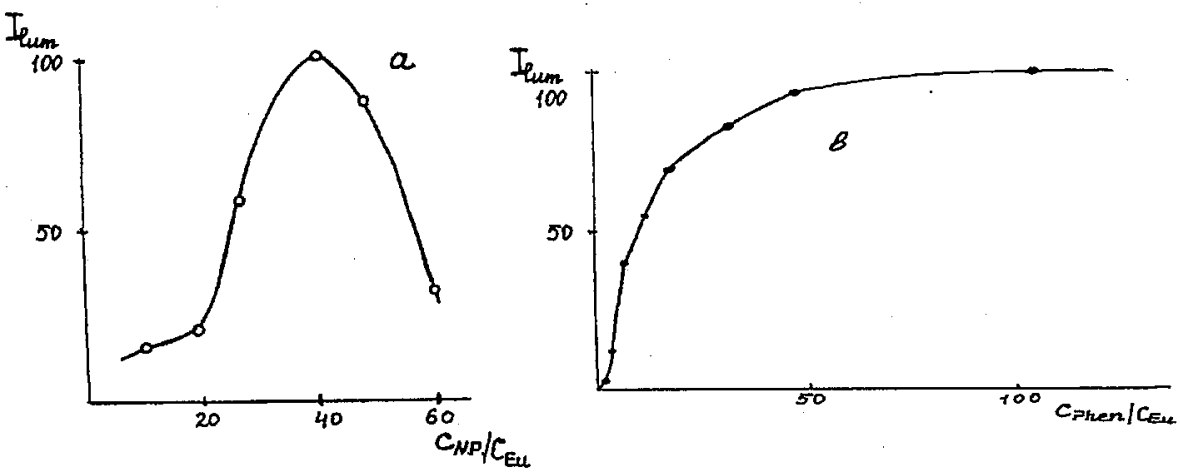

Fig. 6. Dependence of $I_{\mathrm{lum}}$ of $\mathrm{Eu}(\mathrm{III})$ in sorbate of complex on the ligand amount in solution: (a) naphthoic acid; (b) 1,10-phenanthroline; $C_{\mathrm{Eu}}=3 \times 10^{-4} \mathrm{~mol} / \mathrm{L}$.

The dependence of $I_{\text {lum }}$ of $\mathrm{Eu}(\mathrm{III})$ in sorbates on the organic ligand (naphthoic acid and 1,10-phenanthroline) amount is presented in Fig. 6. As can be seen, the highest $I_{\text {lum }}$ of $\mathrm{Eu}$ (III) is observed at 40 -fold excess of naphthoic acid and 100 -fold excess of 1,10-phenanthroline in solution.

\subsection{Influence of surfactants and solvents}

It is known that using the micellar media often causes the increase in $I_{\text {lum }}$ of $\mathrm{Eu}(\mathrm{III})$ in solutions of complexes with organic ligands [7]. In this connection, the influence of cetyltrimethylammonium bromide, cetylpyridinium bromide, triton $\mathrm{X}-100$ as well as the donor-active additive, trioctylphosphinoxide (TOPO), on $I_{\text {lum }}$ of $\mathrm{Eu}(\mathrm{III})$ sorbate was considered. Only cetylrtimethylammonium bromide was found to cause the increase in $I_{\text {lum }}$ of $\mathrm{Eu}$ (III) by 3 times in sorbate. In all the rest of instances the surfactants do not affect $I_{\text {lum }}$ of $\mathrm{Eu}(\mathrm{III})$ in sorbate. In the case of TOPO the decrease in $I_{\text {lum }}$ was observed.

It was also established that when the treatment of zeolite was performed with ethanolic solutions of naphthoic acid and 1,10-phenanthroline, $I_{\text {lum }}$ of $\mathrm{Eu}$ (III) sorbate increased by the order of value. The significant increase in $I_{\text {lum }}$ of $\mathrm{Eu}$ (III) in sorbate (approximately by 20 times) was also observed when the sorbate on zeolite was prepared from benzol (or toluol) extract of $\mathrm{Eu}$ (III) complex with naphthoic acid and 1,10-phenanthroline. It is evident that the quenching action of $-\mathrm{OH}$ bonds 
of water molecules residing in the zeolite cavities was eliminated in the presence of the solvents (ethanol, benzol).

The thermic stability is an important parameter for the materials used as the lamp luminophores. Therefore, the europium sorbates underwent temperature action for $2 \mathrm{~h}$ at $50^{\circ} \mathrm{C}, 100^{\circ} \mathrm{C}, 200^{\circ} \mathrm{C}$, and $250^{\circ} \mathrm{C}$. It was found that $I_{\text {lum }}$ of sorbates was constant upon the heating to $50^{\circ} \mathrm{C}$ and $100^{\circ} \mathrm{C}$, at $200^{\circ} \mathrm{C} I_{\text {lum }}$ reduced by $20 \%$, at $250^{\circ} \mathrm{C}$ by $50 \%$.

\section{Conclusions}

The Iuminescence properties of $\mathrm{Eu}$ (III) sorbates with naphthoic acid and 1,10-phenanthroline on zeolite of CaA-type were studied. It was shown that luminescence intensity of sorbates depends on sorption time of Eu(III) ion and its complex with above-mentioned ligands on the zeolite, on $\operatorname{Eu}(\mathrm{III})$ ion and ligand concentrations and acidity of solution. The influence of cetylrtimethylammonium bromide and of some solvents on $I_{\text {lum }}$ of $\mathrm{Eu}(\mathrm{III})$ sorbate has been demonstrated. The results demonstrate that the search for new luminophore materials through the sorption of the lanthanide complexes with naphthoic acid and 1,10-phenanthroline on the zeolite is promising.

\section{References}

[1] M. Lecomte, B. Viana, C. Sanchez, J. Chem. Phys. Phys.-Chem. Biol. 88, 39 (1991).

[2] N. Takayuki, I. Satoshi, I. Susumu, K. Kunihavu, Makromol. Chem. Rapid Commun. 6, 489 (1985).

[3] V.S. Khomenko, T.A. Paulich. Koordinacionnaya Khim. 21, 510 (1995).

[4] C.R. Ronda. J. Alloys Comp. 325, 534 (1995).

[5] M. Bredol, U. Kynast, C.R. Ronda, T. Welker, Europ. Patent 05226627.

[6] V.T. Mischenko, E.I. Tselik, O.V. Koev. Zh. Analit. Khim. 32, 71 (1977).

[7] S.B. Savvin, R.K. Chernova, S.N. Shtykov, Surfactants, Nauka, Moscow 1991, p. 180. 